\title{
An Optimization of Bluetooth Sensor Locations for Origin-Destination in an Urban Network
}

\author{
Ibrahim Khliefat, Ibrahem Shatnawi \\ Department of Civil Engineering, Faculty of Engineering, Al-Balqa Applied University, Al-Salt, Jordan \\ Email:khliefat@bau.edu.jo,ibh982@yahoo.com
}

How to cite this paper: Khliefat, I. and Shatnawi, I. (2017) An Optimization of Bluetooth Sensor Locations for OriginDestination in an Urban Network. Journal of Transportation Technologies, 7, 367375.

https://doi.org/10.4236/jtts.2017.74024

Received: July 20, 2017

Accepted: August 12, 2017

Published: August 15, 2017

Copyright (๑) 2017 by authors and Scientific Research Publishing Inc. This work is licensed under the Creative Commons Attribution International License (CC BY 4.0). http://creativecommons.org/licenses/by/4.0/

\begin{abstract}
This paper develops an optimization model that determines the optimal location for Bluetooth nodes used in the determination of the origin-destination matrix within an urban network. To analyze the effectiveness of the model, the city of Akron, $\mathrm{OH}$ was utilized as a testing location. The Average Daily Traffic (ADT) was used to determine intersections that have the greatest number of Bluetooth responses. Along with maximizing the number of Bluetooth responses, the model applies financial constraints when determining the number of nodes in the urban network. The developed model selected seven locations to deploy nodes in order to stay within the financial constraints and to maximize the possible number of responses from vehicles within the Akron urban network.
\end{abstract}

\section{Keywords}

Origin-Destination, Bluetooth Technology, Node, Optimal Number

\section{Introduction}

Bluetooth technologies have been widely used in traffic data collection due to its cost effectiveness for travel time estimation. Using this technology, data can be easily collected and processed in a shorter amount of time compared to other conventional methods. Each Bluetooth-enabled device has a unique Media Access Control (MAC) address associated with it. By placing multiple Bluetooth sensors along a corridor, the vehicle speed, travel time and origin-destination data can be collected by matching MAC addresses on multiple nodes. When a vehicle with a detectable Bluetooth device passes a Bluetooth sensor, the MAC 
address and the time of detection are recorded by the sensor. Each MAC device has a unique address which makes it possible to track a vehicle with enabled Bluetooth device via its identification at upstream and downstream points and the time elapsed between these two points can be used to estimate the travel time [1].

The majority of the studies using Bluetooth for traffic data collection focus on vehicle speed and travel time, however, using this technology for origin-destination studies is a relatively new concept. The proposed study aims to develop a methodology for determining the optimal numbers and locations for Bluetooth stations on urban road network using the ArcGIS software. The Geographical Information System (GIS) is used for collecting, analyzing and handling geographically referenced data. A literature review of general O-D matrix methods and Bluetooth O-D methods was conducted to provide the context for a Bluetooth field deployment and implementation. The study presents the deployment plan and data analysis for a hypothetical case study that will be conducted for different urban road network sizes (number of links and junctions).

\section{Objectives}

The main objective of this research project is to develop a system-wide deployment strategy for a Bluetooth implementation in urban road network for O-D estimation. This will be expanded in this research by adding several main arterials and crossroads to the study zone. Another objective is to determine the optimal number of Bluetooth nodes required for the study area.

\section{Background}

Bluetooth technology is typically utilized in research to measure travel times and vehicle speeds. However, we applied this technology to determine the route choice and the origin-destination trips. Bluetooth technology is defined herein as a telecommunications that can be used as a monitoring vehicular and pedestrian traffic in cities. This technology is usually embedded in vehicles, Global Positioning Systems (GPS) and mobile phones.

A team of engineers from the Indiana Department of Transportation used Bluetooth to track travel times for vehicles and pedestrians. This group used Bluetooth technology to estimate waiting time at the airport security line. Many methods were used to estimate travel time based on the collection of timestamped MAC addresses from Bluetooth [2]. The results indicate that the quantity and the quality of the MAC addresses read with each antenna type are different when compared to traffic volumes collected via inductive loop detectors over the same time period Bluetooth is embedded with different technology devices which allow the system to work efficiently with low cost. Therefore, the application of Bluetooth technique in collecting travel times on urban roadways can be carried out programmatically by public sector organizations that need travel time information. It has been shown that Bluetooth technology may be 
deployed at a low cost and result in very effective travel time and speed data for a variety of operation and planning purposes [3]. The usage of innovative techniques to measure traffic data has increased proportionally with technological advancements. With the rise in cell phone usage and the predominance of Bluetooth, methods have been developed and implemented to collect traffic data from roadside sensors. Bluetooth nodes are comprised of a Bluetooth antenna which records the MAC address of a passing cell phone or other Bluetooth enabled devices. The individual node communicates with a server which processes the data collected from multiple nodes placed in the field. The server will group multiple MAC hits of the same device. Then, the hits are matched on multiple nodes to determine the travel time and speed of the same vehicle. Bluetooth nodes determine the space mean speed of vehicles or the average speed over a given distance. The most common application of Bluetooth nodes is in the determination of travel time and speed on roadways. A study conducted along Interstate 95 between Baltimore, MD and Washington DC found that accurate travel times and speeds may be recorded using Bluetooth technology [4]. Another study was conducted to compare travel times recorded on interstates and arterial corridors and found that data from arterials have a larger variance due to traffic signals and the increased number of route options [5]. The nature of matching algorithms and the accuracy of the speeds collected from Bluetooth nodes are evaluated to determine the effectiveness of the filtering system for processing travel times [6]. Haghani, Ali, et al. studied the optimal placement height of the Bluetooth antenna by comparing the number of hits when the mounting height is varied from zero to ten feet above the roadway surface and their results recommended mounting the antenna at least eight feet above the roadway [7].

Recently, researchers have been experimenting the usage of Bluetooth nodes in origin-destination (O-D) studies. The resulting O-D matrices developed from Bluetooth nodes have been compared to O-D data from a video and an automated number plate recognition and the results indicate that the data from Bluetooth is similar to the traditional methods [8]. Data from Bluetooth nodes have been also used to estimate O-D matrices using an ad hoc linear Kalman filter approach by conducting a computational experiment with a set of simulated traffic data [9]. In addition, a study in Jacksonville, FL placed Bluetooth nodes along arterial and competing routes to determine route specific O-D tables [10].

\section{Concept of Bluetooth Technology}

The application of Bluetooth technology is limited for short-range wireless communications and is used to determine vehicle travel time and speed between the passed points on a roadway network. Travel time measurement and estimation are important issues for real-time traffic management. To estimate travel time of a corridor using Bluetooth technology, MAC addresses, and time stamps are generally filtered and matched. Once a Bluetooth-enabled device travels 
along a roadway, its unique MAC address as well as its location and real timestamp are recorded by a roadside device. When the same MAC address is recorded at different points on a roadway segment of a known distance, the travel time can be calculated as the difference between the recorded timestamps at those corresponding points.

The system architecture components which are required to estimate vehicle travel time via using Bluetooth devices are:

- "Bluetooth system on a roadside to detect and process MAC address as the vehicles travel along the monitored corridor;

- A radio that can read the MAC address within the communication range for detection; and

- A Central Processing Unit (CPU) which is placed in the site that has a special software for detecting, processing, and forwarding the information to a central location".

Figure 1 [5] shows a diagram of the components required and the concept used in the Bluetooth Traffic Monitoring Concept.

\section{Optimization Model}

This study utilized Sherali [11] model to determine the optimal number of locations to place the Bluetooth stations to capture the O-D Pairs. In the proposed mathematical model, we denote nodes as $N$ and links as $L$. The objective function can be derived from the multiplication of traffic flow of each link $(L)$ and the variation of traffic on that link is denoted as LCOV. The purposes of the objective function are as follows:

1) To minimize the cost associated with installing Bluetooth stations (fiscal constraint);

2) To maximizes the traffic flow being detected in the network.

Illustrating a network as $G(N, L)$, as previously mentioned $N$ is nodes, and $L$ is links as shown in Equation (1)

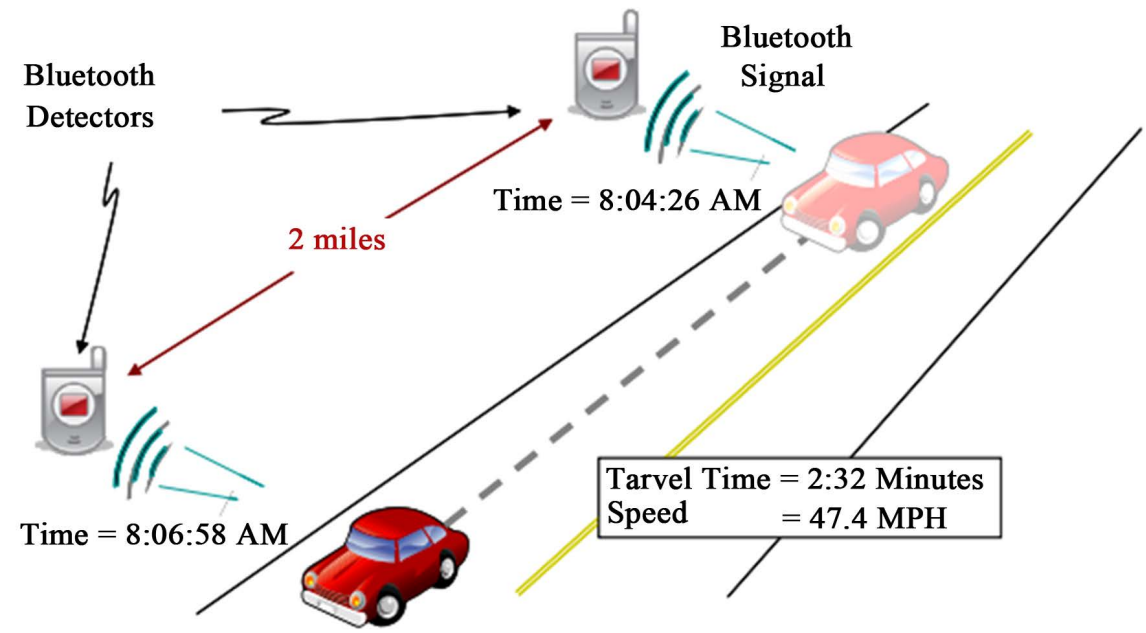

Figure 1. Bluetooth traffic monitoring concept. 


$$
b_{i j}=t f_{i j} L C O V_{i j}, \forall(i, j) \in L
$$

where:

$b_{i j}$ : Benefit factor for covering arc $(i, j) \in L$;

$t f_{i j}$ : Traffic flow on $\operatorname{arc}(i, j) \in L$;

$L C O V_{i j}$ : Link coefficient of variation of traffic flow on arc $(i, j) \in L$;

$C_{j}$ : Cost associated by installing a Bluetooth station at location $j ;$

$B$ : Budgetary constraint;

$R$ : Maximum number of available Bluetooth;

$(i, j)$ : Origin-Destination (O-D) Paris.

Decision Variable is represented using Equation (2):

$$
y_{j}:\left\{\begin{array}{cc}
1, & \text { if a bluetooth located at node } j \\
0, & \text { Otherwise }
\end{array} ; j \in L\right.
$$

The objective function can be formed as shown in Equation (3):

$$
\max \sum \sum b_{i j} y_{i} y_{j}
$$

S.t.

$$
\sum_{j \in N} y_{j} \leq R
$$

The scan radius for Bluetooth is 750 feet $\geq L D \geq 500$ feet

$A D T_{n} \geq 4000$ Average daily traffic, directional volume for intersection approach

where:

$L D$ : Link distance between two nodes;

$A D T_{n}$ : Average daily traffic at a particular node.

$$
\begin{gathered}
\sum_{j \in N} C_{j} y_{j} \leq B, y \in(0,1) \\
\max \sum \sum b_{i j} y_{i} y_{j}
\end{gathered}
$$

is seeks to maximize.

$$
\sum_{j \in N} y_{j} \leq R
$$

Number of Bluetooth should not exceed the available maximum number $R$.

Budget constraints and Logical binary restrictions on decision variables can be represented by Equations (4) and (5) respectively.

$$
\begin{gathered}
\sum_{j \in N} C_{j} y_{j} \leq B \\
y \in(0,1)
\end{gathered}
$$

A flow chart showing the flow of the optimization model and ArcGIS interface is shown in Figure 2.

The model may need to be executed through several iterations to get the optimal results. The flow chart presents a process that may be followed to determine the optimal node locations within an urban network.

\section{Study Area}

This research focuses on an urban area for the Bluetooth technology deploy- 
ment. Accordingly, Akron Central Business District (CBD) in Summit County, $\mathrm{OH}$ has been selected for determining the optimal locations of Bluetooth nodes in the urban road network for estimating O-D matrices. Land use in this area primarily consists of urban core and suburban city development including extensive commercial, industrial and residential development. Figure 3 shows the Average Daily Traffic (ADT) for the City of Akron.

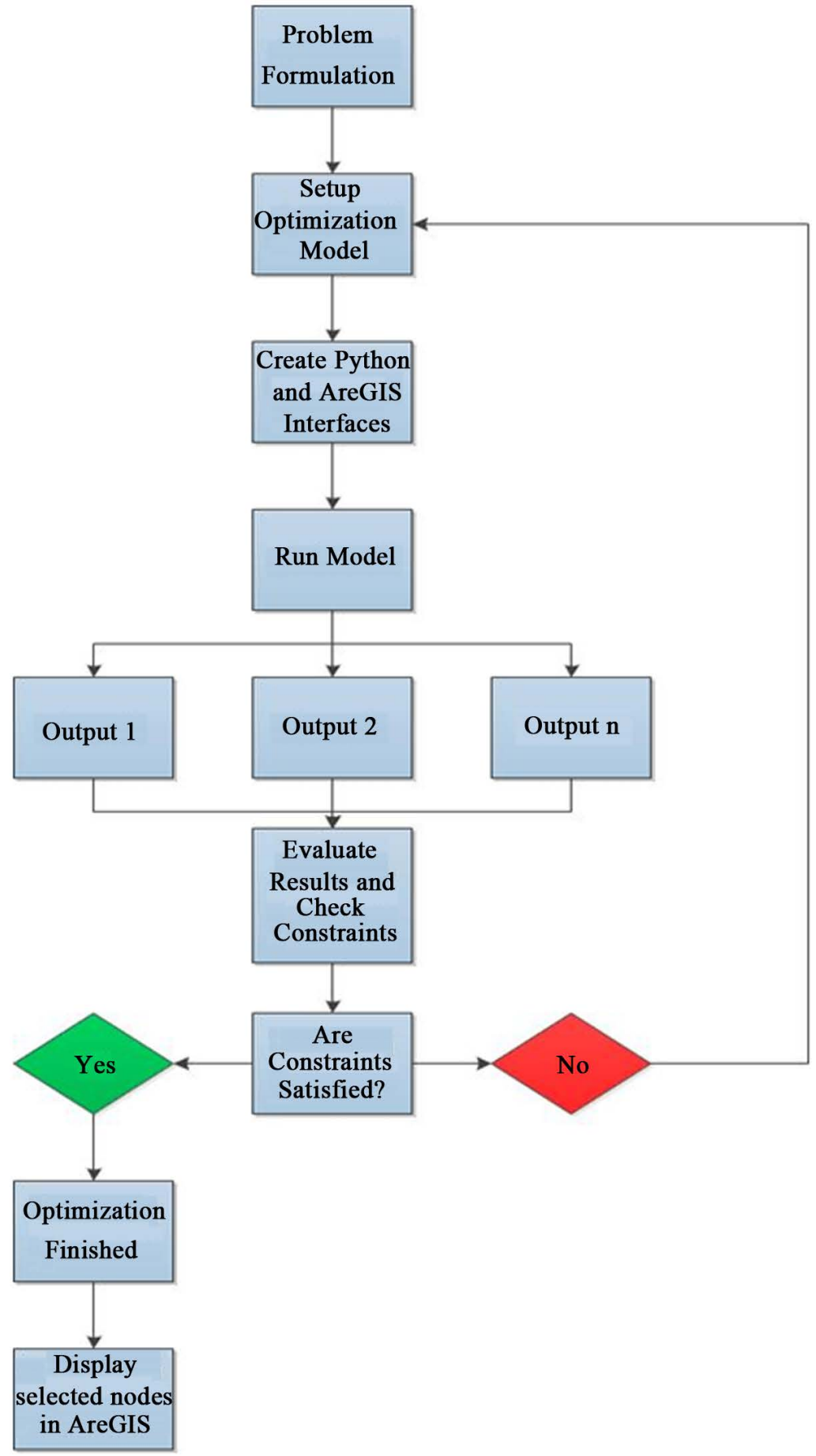

Figure 2. The flow of optimization model. 


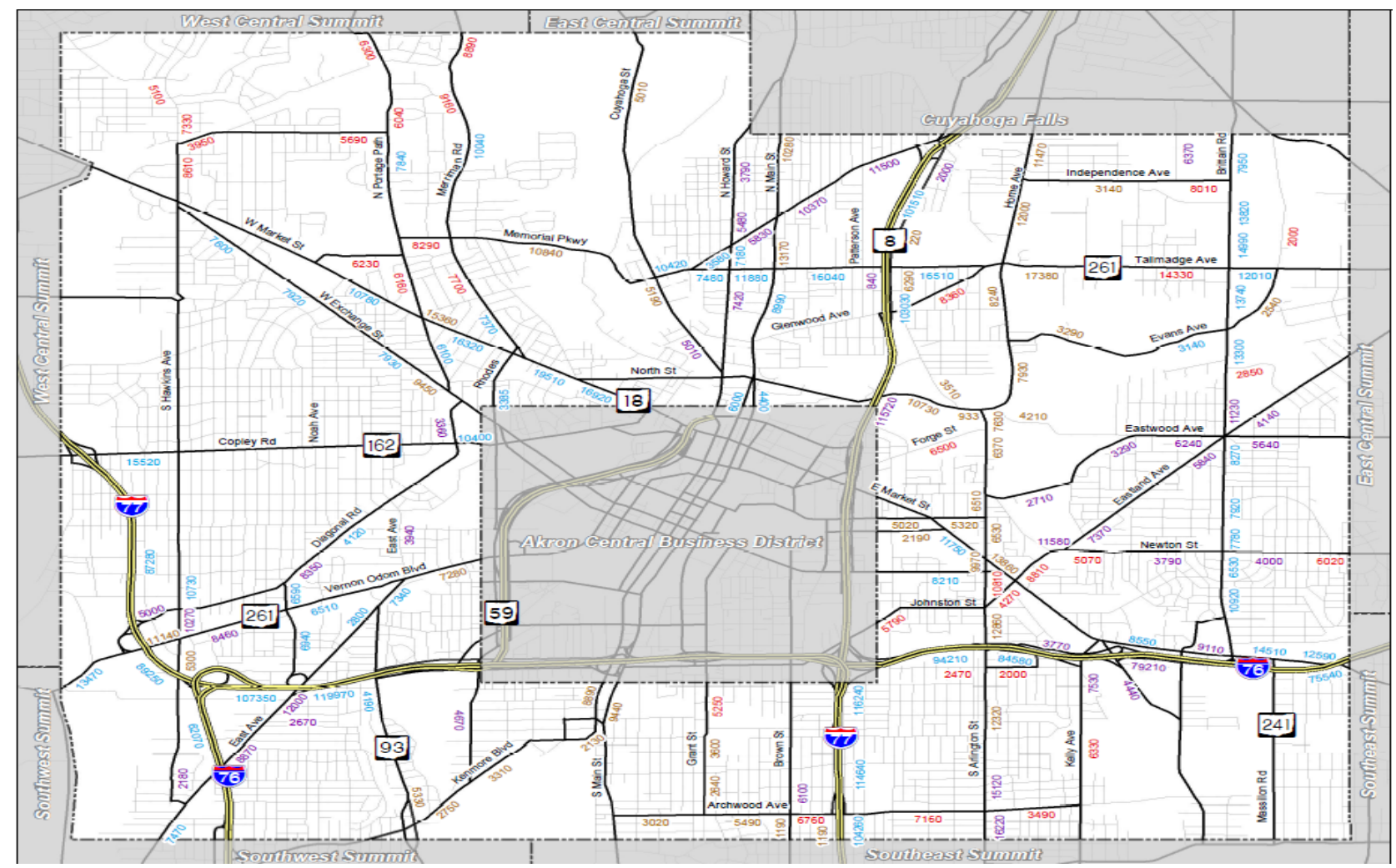

Figure 3. Average daily traffic for the city of Akron.

\section{Implementation}

An optimization model has been developed to determine the optimal number of Bluetooth nodes required in a given network. The model considers the geographical size of the network and cost constraints associated with implementing this technology. However, the developed optimization model has some constraints such as the scan radius of Bluetooth nodes and the ADT at intersections. The optimization model is discussed in more detail in the following section of the paper. The urban road network for Akron has been coded using Python within the ArcGIS platform. Using GIS tools, the traffic volume for road segments were inserted in the Akron map layer. The total ADT's at intersections have been calculated from the incoming links between the nodes. Figure 4 shows the process of the GIS modeling.

Scripting in Python was done to create and automate GIS workflows quickly and easily. An interface between GIS platform and Python program language has been created to implement the optimization model. The screenshot, which is shown in Figure 5, illustrates how to get the node selected in the layer according the optimization model which has been coded in Python.

\section{Conclusion}

An optimization model has been developed in order to determine the optimal locations for Bluetooth nodes to determine the O-D matrix within an urban 


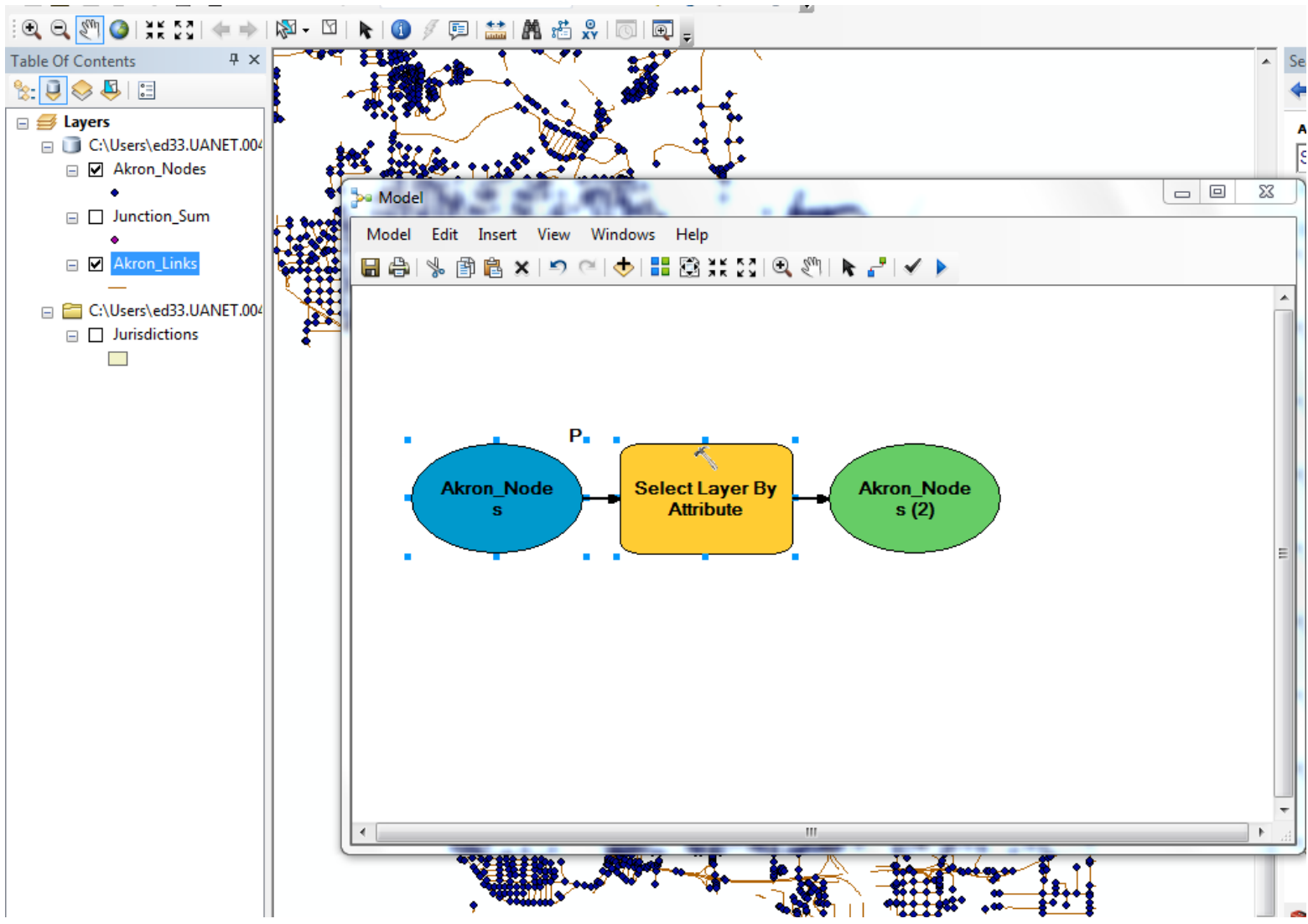

Figure 4. GIS modeling.

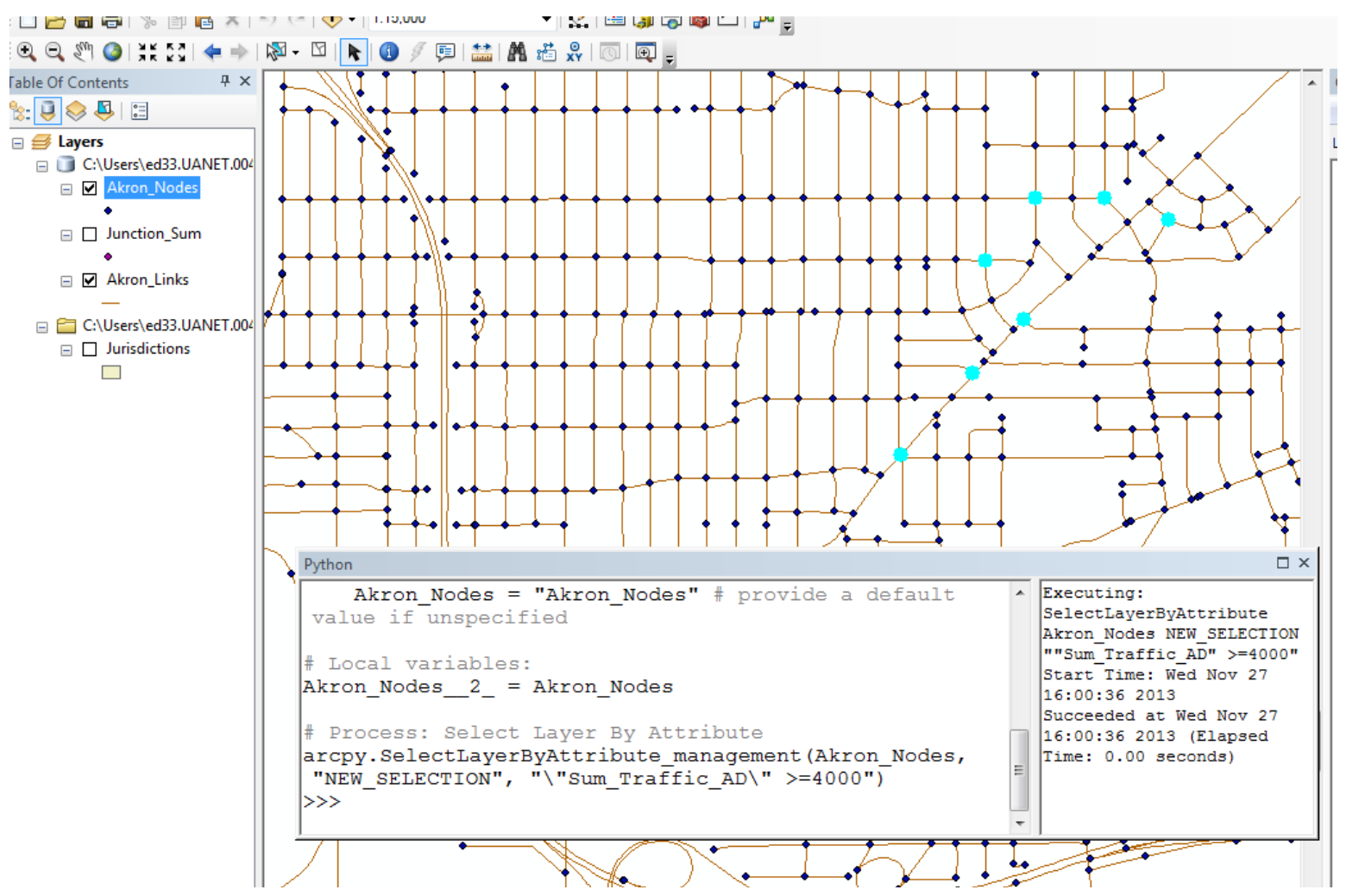

Figure 5. Node selection script. 
roadway network. The model is designed to account for fiscal constraints of an agency implementing the Bluetooth nodes as well as maximizing the volume of traffic that may be detected by the nodes in the network. The model was applied to the CBD in the city of Akron to evaluate the effectiveness of the model in selecting the optimal locations for the Bluetooth nodes. The model selected seven locations to deploy nodes in order to stay within the financial constraints and to maximize the possible number of responses from vehicles within the network.

\section{References}

[1] Araghi, B.N., et al. (2015) Reliability of Bluetooth Technology for Travel Time Estimation. Journal of Intelligent Transportation Systems, 19, 240-255. https://doi.org/10.1080/15472450.2013.856727

[2] Porter, J.D., Kim, D.S., Magaña, M.E., Poocharoen, P. and Arriaga, C.A.G. (2013) Antenna Characterization for Bluetooth-Based Travel Time Data Collection. Journal of Intelligent Transportation Systems, 17, 142-151. https://doi.org/10.1080/15472450.2012.696452

[3] Puckett, D.D. and Vickich, M.J. (2010) Bluetooth ${ }^{\oplus}$-Based Travel Time/Speed Measuring Systems Development (No. UTCM 09-00-17).

[4] Young, S.E. (2012) Bluetooth Traffic Detectors for Use as Permanently Installed Travel Time Instruments (No. MD-12-SP909B4D).

[5] Young, S. (2008) Bluetooth Traffic Monitoring Technology. http://www.catt.umd.edu/sites/default/files/documents/UMD-BT-Brochure_REV3. pdf

[6] Haghani, A., Hamedi, M., Sadabadi, K., Young, S. and Tarnoff, P. (2010) Data Collection of Freeway Travel Time Ground Truth with Bluetooth Sensors. Transportation Research Record: Journal of the Transportation Research Board, 2160, 60-68. https://doi.org/10.3141/2160-07

[7] Brennan Jr., T.M., Ernst, J.M., Day, C.M., Bullock, D.M., Krogmeier, J.V. and Martchouk, M. (2010) Influence of Vertical Sensor Placement on Data Collection Efficiency from Bluetooth MAC Address Collection Devices. Journal of Transportation Engineering, 136, 1104-1109. https://doi.org/10.1061/(ASCE)TE.1943-5436.0000178

[8] Blogg, M., Semler, C., Hingorani, M. and Troutbeck, R. (2010) Travel Time and Origin-Destination Data Collection Using Bluetooth MAC Address Readers. Proceedings of the Australasian Transport Research Forum 2010, Canberra, 29 September-1 October 2010, 1-15.

[9] Barceló, J., Montero, L., Marqués, L. and Carmona, C. (2010) Travel Time Forecasting and Dynamic Origin-Destination Estimation for Freeways Based on Bluetooth Traffic Monitoring. Transportation Research Record: Journal of the Transportation Research Board, 2175, 19-27. https://doi.org/10.3141/2175-03

[10] Carpenter, C., Fowler, M. and Adler, T. (2012) Generating Route-Specific OriginDestination Tables Using Bluetooth Technology. Transportation Research Record: Journal of the Transportation Research Board, 2308, 96-102. https://doi.org/10.3141/2308-10

[11] Sherali, H.D., Desai, J. and Rakha, H. (2006) A Discrete Optimization Approach for Locating Automatic Vehicle Identification Readers for the Provision of Roadway Travel Times. Transportation Research Part B: Methodological, 40, 857-871. https://doi.org/10.1016/j.trb.2005.11.003 